\title{
TOWARD THERMODYNAMICS OF REAL-TIME SCHEDULING
}

\author{
I. MAYOROV ${ }^{1} \&$ P. SKOBELEV ${ }^{2,3}$ \\ ${ }^{1}$ Samara State Technical University, Russia. \\ ${ }^{2}$ Smart Solutions, Ltd, Russia. \\ ${ }^{3}$ Samara State Aerospace University, Russia.
}

\begin{abstract}
The modern problem of real-time resource management to increase enterprise efficiency is considered.

A new look at the dynamic self-organizing processes based on multi-agent technologies in building and revising schedules by events in real time is suggested. Schedule is considered as a flexible network of operations of demand and resource agents. This schedule is formed during the interactions of basic agent classes that set and break the dynamic links between each other, depending on the events and changing situation in the real world.

A thermodynamic model of demand-resource network (DRN) dynamics is introduced. There is a similarity to Ilya Prigogine's non-linear thermodynamics theory which allows us to explain the phenomenon of unstable equilibrium emergence, order and chaos, catastrophes, bifurcations and other non-linear events that are significant to the self-organizing processes control in multi-agent systems (MASs).

Keywords: adaptability, chaos and order, complex systems, demand-resource network, multi-agent technology, network dynamics model, non-equilibrium, real-time scheduling, self-organizing.
\end{abstract}

\section{INTRODUCTION}

Global economy's complexity is well known nowadays, which is often driven by uncertainty and dynamics in demand and supply.

Under these circumstances, the more operative, flexible and efficient approaches to the decisionmaking for resources allocation, scheduling, optimization, coordination and control are needed. These approaches allow the enterprises to maintain their profitability by avoiding equipment downtime or shortages of all kinds of resources, including personnel, equipment, finance and others. Therefore, the idea of a 'real-time smart enterprise' is discussed more and more often. These enterprises will have 'on-the-fly' dynamic resources scheduling in real time, since decisions' quality and efficiency directly depend on the moment of time itself. Moreover, in the conditions of today's competitive environment, delayed decisions lead to the losses.

A new approach to scheduling is suggested in this paper. In this approach, resources schedule is not a static data structure that is created only once and becomes outdated immediately, but it is considered as a dynamic self-organized data structure that adaptively changes. Unlike our earlier papers dedicated to the DRN creation for adaptive intelligent resource scheduling system based on the multi-agent technologies [1,2], in this paper we suggest and justify a new 'thermodynamic' interpretation of self-organization processes of scheduling that shows a deep analogy of these processes to the non-linear thermodynamics suggested by Prigogine and Stengers [3] and Prigogine and Nicolis [4].

There is a gap between the existing solutions built based on the combinatorial method and scheduling tools [5], where we need to know all the orders and resources beforehand. Traditional systems implement batch versions of linear or dynamic programming, constraint programming and other methods based on combinatorial search of options [6,7], which in practice turn out to be unsuitable. In contrast with the classical large, centralized, monolithic and sequential programs, multi-agent

(C) 2015 WIT Press, www.witpress.com

ISSN: 1755-7437 (paper format), ISSN: 1755-7445 (online), http://www.witpress.com/journals DOI: $10.2495 / \mathrm{DNE}-\mathrm{V} 10-\mathrm{N} 3-213-223$ 
systems (MASs) are built as distributed communities of small autonomous software objects working asynchronously but in a coordinated way to obtain the results.

Multi-agent technology is considered as a new paradigm for solving complex problems that are difficult or even impossible to solve by classical mathematical methods or algorithms [8], for example, in scheduling and optimization, pattern recognition, text understanding and some other domains. This paradigm is considered as one of the most innovative and efficient for real-time scheduling of resources 'on the fly' $[9,10]$.

In this paper, we will consider the basic concepts of the approach and show its applications as well as a direct link with models of thermodynamics.

\section{DEVELOPMENT OF THE CONCEPT OF DRN}

The concept of DRN was suggested in our early papers on multi-agent approach. This concept was successfully applied in the creation of a gallery of adaptive real-time schedulers [1,2,7].

The DRN model is based on a 'holonic' approach to the production systems creation [11] where specific classes of agents are introduced.

As a part of the holonic approach in the DRN, we introduce demand and supply agents. These agents can act as sub-agents (assistants) to the main agents listed above, allowing them to concurrently or asynchronously search for each other on the virtual market, performing a continuous matching (Table 1).

A constant matching between the competing and cooperating demand and supply agents on the system's virtual market allows to build a solution to any complex problem as a dynamic network of connections between tasks (operations) that is easily modified in real time.

In the DRN concept, any physical or abstract entity agent of problem domain can create agents of demand or resources. As a result, the schedule can be formed as a kind of requirement-driven network of operations, which can be easily adapted by events in real time [1,2].

Continuous improvement of the system key performance indicators is based on the compensation method, where agents, whose condition became worse, receive compensation in the form of virtual money from agents that improved their schedule.

Specific DRN-based methods and tools were developed to design adaptive MASs for real-time scheduling [12].

\subsection{Thermodynamic DRN scheduling model}

Even a small organization schedule is a much more complex and dynamic object than it may seem at first glance because self-organizing processes that are similar to the ones described in Ilya Prigogine's writings began to play an increasing part in scheduling in our systems.

In general terms, it is suggested to consider the schedule of a complex system as 'unstable equilibriums', which differ in strength in different directions. A new order brings in money that plays the part of the systems energy. Order and chaos phenomena, autocatalytic reactions, oscillations and other non-linear events arise in the system. If the conflicts resolution and the number of messages in the system increase, we can assume that the temperature of the schedule section is increasing as well. Number of messages related to number of nodes plays the role of the system temperature. To support the structure of schedule, agents are paying some taxes that dissipate part of the input energy.

If orders stop coming in, this schedule will begin to gradually disintegrate in some time, from the weakest links between agents to the system transition to a state of complete chaos with zero energy, and cool down completely. 
Table 1: Main classes of agents.

\begin{tabular}{|c|c|c|}
\hline Agent class & $\begin{array}{l}\text { Specification of agent behavior, main } \\
\text { goals and tasks }\end{array}$ & Attributes \\
\hline Order agent & $\begin{array}{l}\text { The goal of the order agent is to complete } \\
\text { the order in time with maximum quality, } \\
\text { minimal cost, best delivery time and } \\
\text { minimum risk. Creates operation agents } \\
\text { corresponding to the technological } \\
\text { operations or business processes }\end{array}$ & $\begin{array}{l}\text { Defines service level, real and } \\
\text { virtual money balance for order } \\
\text { execution, given the specifications } \\
\text { for resources, operations } \\
\text { interdependencies, deadline for } \\
\text { order execution, risks }\end{array}$ \\
\hline $\begin{array}{l}\text { Product } \\
\text { (result) agent }\end{array}$ & $\begin{array}{l}\text { The goal is to get the best result to match } \\
\text { order specifications and requirements }\end{array}$ & $\begin{array}{l}\text { Domain-specific product } \\
\text { requirements which are specified in } \\
\text { order }\end{array}$ \\
\hline $\begin{array}{l}\text { Resource } \\
\text { agent }\end{array}$ & $\begin{array}{l}\text { The goal is to maximize resource } \\
\text { workload, get the best orders, tightly } \\
\text { schedule the executed operations or } \\
\text { provide continuous operation and } \\
\text { specified requirement execution }\end{array}$ & $\begin{array}{l}\text { Availability at any moment of time, } \\
\text { scale of charges of usage, working } \\
\text { capacity and maintenance, efficiency }\end{array}$ \\
\hline $\begin{array}{l}\text { Operation } \\
\text { agent }\end{array}$ & $\begin{array}{l}\text { Coordinates performance of specific } \\
\text { technological operations and business } \\
\text { processes, their scheduling on resources, } \\
\text { searches for the best resources for } \\
\text { execution, negotiates about sub-tasks } \\
\text { allocation, proactively improves the } \\
\text { situation in the schedule }\end{array}$ & $\begin{array}{l}\text { Preferable and real allocation time, } \\
\text { virtual and real accounts, current } \\
\text { penalties and bonuses, processes and } \\
\text { operations deadline }\end{array}$ \\
\hline $\begin{array}{l}\text { Staff agent } \\
\text { Or enterprise } \\
\text { agent }\end{array}$ & $\begin{array}{l}\text { Controls enterprise key performance } \\
\text { indicators and balance workload of the } \\
\text { resources, discovers the 'bottlenecks' in } \\
\text { the schedule and generates overcoming } \\
\text { strategies, seeks to increase profit and } \\
\text { other specified indicators }\end{array}$ & $\begin{array}{l}\text { List of available resources, } \\
\text { preferences and constraints for this } \\
\text { task or organization }\end{array}$ \\
\hline $\begin{array}{l}\text { Demand } \\
\text { agent }\end{array}$ & $\begin{array}{l}\text { Allows to find a supply (resource) by } \\
\text { the requirement, using requirements } \\
\text { specification }\end{array}$ & $\begin{array}{l}\text { List of situational matching } \\
\text { parameters }\end{array}$ \\
\hline Supply agent & $\begin{array}{l}\text { Allows to find a demand by the } \\
\text { requirement, using requirements } \\
\text { specification }\end{array}$ & $\begin{array}{l}\text { List of situational matching } \\
\text { parameters }\end{array}$ \\
\hline
\end{tabular}

\subsection{Satisfaction and formal problem statement description}

The formalized problem statement is based on searching for a consensus between agents in DRN virtual market and can be formulated as follows.

Let us assume that all agents of demands and supply have their own goals, criteria, preferences and constraints (e.g. due date, cost, risk, priority, required equipment type or worker qualification). The importance of each criterion can be represented by weight coefficients in a linear combination of criteria for the given situation in scheduling, but can change during the schedule forming or execution. 
(a)

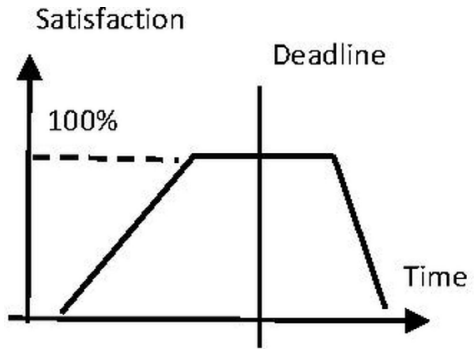

(b)

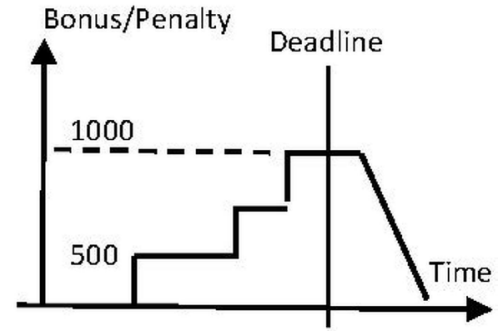

Figure 1: Example of (a) satisfaction function and (b) bonus/penalty function.

Let us introduce the satisfaction function for each agent (Fig. 1a), which will show deviation of the current value of this function from the given ideal value by any of the criteria for the current step of finding scheduling solution for this agent. The activity of agents also depends on bonus/penalty function and current budget allocated on specific accounts for virtual money (Fig. 1b).

Let each demand $j$ has several individual criteria $x_{i}$ and suggested ideal values $x_{i j}^{i d}$. For each agent of demand $j$, a normalized bonus/penalty function is calculated by component $I$, given, for example, as a linear function $f_{i j}^{t a s k}\left(x_{i}-x_{i j}^{i d}\right)$. In most cases, this function has a bell form with maximum in the point of suggested ideal value. After that, a satisfaction value for each task agent and each criterion $i$ with the given weight coefficients $a_{i j}^{\text {task }}$ task is estimated.

By the proper selection of signs and form of the function, the goal of agent system can be reformulated as maximizing of virtual value $y_{j}^{\text {task }}$ of demand $j$ (upper index task means that the values belong to the demand agents):

$$
y_{j}^{\text {task }}=\sum_{i} \alpha_{i j}^{\text {task }} \cdot f_{i j}^{\text {task }}\left(x_{i}-x_{i j}^{i d}\right)
$$

where $\forall j$ weight coefficients are normalized: $\Sigma_{\mathrm{i}} a_{i j}^{\text {task }}=1$.

Similarly, the problem of finding the states $x_{i j}^{*}$ of agents of demands $j$ that maximize the total value of all orders can be formulated:

$$
\begin{aligned}
y^{\text {task }} & =\sum_{j} \beta_{j}^{\text {task }} y_{j}^{\text {task }}=\sum_{j} \beta_{j}^{\text {task }} \sum_{i} \alpha_{i j}^{\text {task }} f_{i j}^{\text {task }}\left(x_{i}-x_{i j}^{\text {id }}\right) \\
y^{\text {task }} & =\max _{x i}\left(y^{\text {task }}\right)
\end{aligned}
$$

where $\beta_{j}^{\text {task }}$ is demand weight that allows to set and dynamically change the priorities showing the importance of criteria.

Similarly, the value function can be given for the supply by criteria $z_{k}$, with bonus/penalty function $f_{k l}^{\text {res }}\left(z_{k}-z_{k l}^{i d}\right.$ ), weight $\alpha_{k i}^{\text {res }}$ of criterion $k$ for resource $l$, and resource value $\beta_{i}^{\text {res }}$ for the system (which is similar for weight for demand agents function):

$$
\begin{aligned}
& y^{r e s}=\sum_{i} \beta_{i}^{r e s} \cdot y_{i}^{r e s}=\sum_{i} \beta_{i}^{r e s} \sum_{k} \alpha_{k i}^{r e s} \cdot f_{k i}^{r e s}\left(z_{k}-x_{k i}^{i d}\right) \\
& y^{r e s^{*}}=\max _{z k}\left(y^{r e s}\right), \\
& z_{k} \in D^{k}, x_{i} \in D^{I} \forall i, k, I=\operatorname{Dim}\left(D^{I}\right), K=\operatorname{Dim}\left(D^{k}\right) .
\end{aligned}
$$


Variables $x$ and $z$ belong to some areas of the space of criteria for demands and supply, $I$ and $K$ are dimensions of the corresponding criteria spaces, and upper index res means that the values belong to resource agents.

Thus, in DRN, the optimization problem is formulated as solving eqns (2)-(4).

In other words, in the suggested bottom-up methodology, one global optimizer is replaced by many small local optimizers which are able to negotiate and find trade-offs when they search their local optimums.

The process of computations will be stopped and solution of problem will be delivered when consensus between agents is found.

\subsection{DRN dynamics}

Fundamental principles that form the basis of a new thermodynamic model of a DRN are the following:

- Each of our systems is open, which means that new orders and resources arrive at any moment of time, causing new agents' appearance and changes in the internal configuration of the connections between them in the system.

- Each new order brings real money into the system that plays the part of 'energy' required for the support of agents' activity (dynamics) and reconnection (statics), and part of this energy dissipates (stored in the system).

- Each agent is given a satisfactory function as its objective function, which shows how close the agent is to the specified ideal values by a certain criterion or their convolution, to which the agent converges.

- The further the agent is from the ideal value, the greater is the agent's 'aspiration' to achieve the results, the more active is an unsatisfied agent and the less active is a satisfied agent. This allows to control agents' internal activity in the system, which is particularly required for complex schedules design with hundreds of thousands agents.

- Agents are awarded if their state improves toward the set goal and get penalties otherwise. For that purpose, a special microeconomics of considered systems, where agents receive virtual currency and pay for a position in the schedule, changes in schedule or communication with other agents and taxes for the specified activity types. This is a dissipative part of the system.

- Each agent has a current virtual account and financial resources that are used by agents to improve their local allocation in the schedule.

- Agents iteratively improve their criteria in order to reach the best values that are close to ideal, compensating other agents' losses in concessions from their budget.

DRN model can be a powerful tool for self-organization processes' description and research in MAS.

It is convenient to introduce the following concepts for new system phenomena description connectivity, connection strength and autonomy level [13].

The connectivity of agents denotes the degree to which an agent is connected to other agents in the system. The higher the connectivity of agents, the greater is complexity of the system.

The connectivity of agents can flexibly change during the computations and at first, it limits agent choices by the closest environment, but the local interaction area is gradually growing unless there is no better solution. 
In fact, it allows to control the speed and quality of a problem solution, increasing or reducing opportunities for self-organization in the system. Connection strength between agents denotes the mutual agent's satisfaction of the connection and degree of breakability of connections.

Weaker connections are easier to break and make new connections. Obviously, connection strength between agents can be calculated as an average satisfaction with connection. That causes misbalance of connections and that increases complexity and, therefore, the unpredictability of global behavior. The weaker the inter-agent connection, the greater is complexity of the system. Similar to the thermodynamics theory, the easier connections and, therefore, the more flexible the system is, the closer it is to the chaos and vice versa, with stronger connection between the agents system reaching a strict order state. Autonomy of agents denotes the degree of freedom given to them to decide what to do. The higher the autonomy of constituent agents, the greater is complexity of the system.

DRN model, topology and connection strength allow us to introduce order and chaos characteristics in MAS.

Lesser the connections, more is the chaos, and the more the connections and their strength, the higher is order degree. A number of connections of one agent can be used for order degree evaluation. Quantitative characteristics and their dynamics are the subject to our future investigations.

Demand agents establish links with resource agents using virtual money as payment for connection. If it is necessary, other agents' deteriorations can also be compensated with virtual money. Therefore, virtual money in such processes represent the energy that agents can use to improve their states. After the rescheduling, agents have some money left and the proportion of free energy decreases. Over time, agents' possibilities to change the schedule with compensations decrease, connection costs increase and their relevance in the sense of a positive impact on the degree of satisfaction of the system is lost. Staff agent can determine which of the connections only inflict losses and that it is energetically profitable to destroy them and plan the costly schedule parts. Therefore, some schedules 'crush' at some point.

New order arrival brings in free energy for the schedule changes. Schedules deterioration can be caused by the energy (virtual money) dissipation in the system. That corresponds with the open systems behavior in Prigogine's thermodynamics.

The connectivity, connection strength and autonomy of agents provide an opportunity to consider the evolution of interacting agents in the DRN using thermodynamic description. This description is similar to the autocatalytic reactions' description in complex multicomponent systems.

\section{SELF-ORGANIZATION IN COMPLEX SYSTEMS}

The general behavior of complex systems is composed of agents' interactions, which in turn constrain their behavior. This behavior is called emergent, and it is unpredictable, but not chaotic. Emergency leads to the events associated with the dynamic rescheduling, as well as to the system adaptability to the impact of external influences.

\subsection{Measuring adaptability of MASs}

MAS receives orders that are scheduled for execution on resources. Incoming and previously distributed orders are dynamically reallocated to the system resources, since the agents tend to increase their satisfaction.

When a new order arrives and it is not distributed by the system yet, system satisfaction drops as the new agent cannot find the best allocation immediately. Over time, the overall satisfaction increases by rescheduling and gradual improvement of agents' conditions. The system goes into a non-equilibrium state, and then the agents try to find new local balance. It is suggested to calculate 
an average satisfaction for $y$ tasks and resources agents depending on the time, in order to evaluate the dynamics of MAS:

$$
y(t)=\frac{\sum_{j} y_{j}^{\text {task }}(t)+\sum_{I} y_{I}^{\text {res }}(t)}{M(t)+N(t)},
$$

where $y_{j}^{\text {task }}$ is the task $j$ agent satisfaction, $y_{i}^{\text {res }}$ the resource $l$ agent satisfaction, and $N(t)$ and $M(t)$ are the number of task and resource agents correspondingly. The tasks come into the system and the resources can be turned on and off; therefore, their number depends on time.

Let us introduce a degree of adaptability $\gamma$ of an MAS [14]. This degree characterizes the rate of local equilibrium recovery:

$$
\gamma=\left(y_{2}-y_{1}\right) \cdot \frac{1}{T}
$$

where $y_{1}$ is a minimum satisfaction value after the influence, $y_{2}$ is average system agents' satisfaction after the MAS was influenced and $T$ is the time of equilibrium recovery of the average satisfaction $y_{2}$.

A similar effect of a partial recovery can be observed not only when the resources are turned off but also with the abrupt appearance of new task flows. The higher the degree of adaptability, the greater the ability to agents' self-organization in elimination of disruptive influences is.

It is clear that MAS adaptability depends on the external influences intensity; for example, with the arrival of large task flows that exceed system productivity, the satisfaction decline will only grow with time, as the dissatisfaction increase of the arrived agents will not be covered by the partial growth due to rescheduling. Dynamics of the MAS satisfaction recovery during resources disabling in a time equal to 18 points are shown in Fig. 2.

After the average satisfaction maximum decrease to the $y_{1}$ level at time $T$, MAS finds a new quasiequilibrium state $y_{2} . \Delta z=y_{\max }-y_{2}$ is an irretrievably lost satisfaction. Similarly, we can consider task agents adaptability $\gamma^{\text {task }}$ and resources agents adaptability $\gamma^{\text {res }}$.

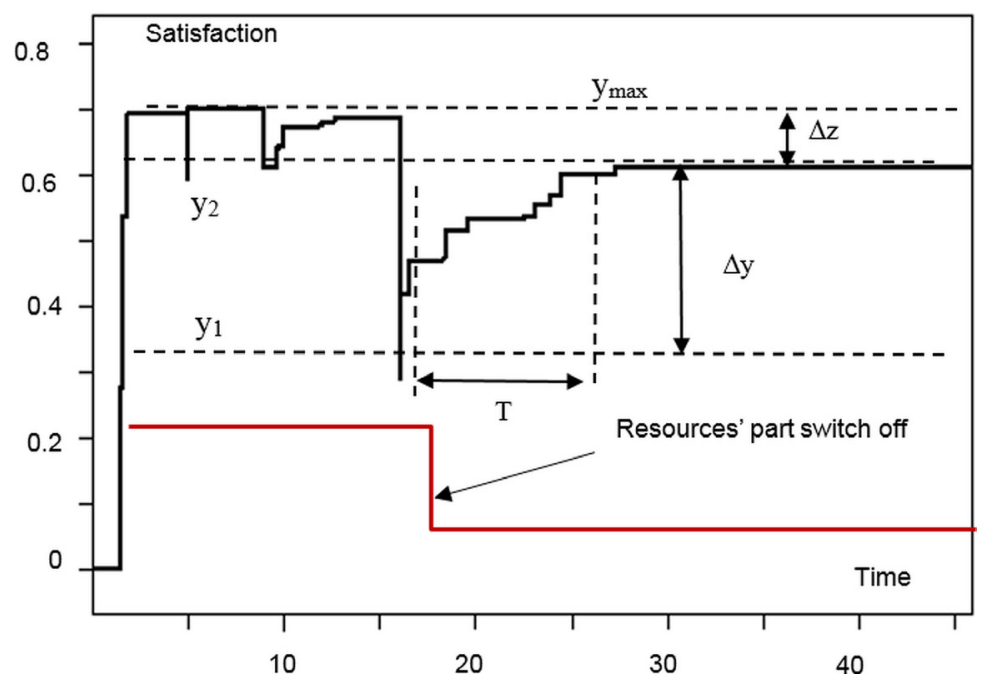

Figure 2: Adaptive partial recovery of the characteristics of a real MAS. 
Therefore, DRN degree of adaptability can reflect the degree of 'adaptive intelligence' as a measure of agents' self-organization.

\subsection{State of unstable equilibrium and disaster}

During their evolution, MASs discover specific events such us dynamic formation of nonequilibrium structures of different depths of inclusion and configuration (like formation of order from chaos) represented by spontaneous acceleration of agent negotiations, leading to a 'constructive destruction' of a schedule in order to create a new schedule of higher quality. Spontaneous acceleration can occur without any apparent cause, akin to autocatalytic processes [4]. The acceleration usually leads to the accumulation of virtual money resulting in a kind of explosion or catastrophe (radical changes in the schedule). Let us consider this on the example of transport logistics problem in Fig. 3.

The scene depicts a community of several trucks, each in turn containing a community of journeys, each of which containing a community of cargoes. Each link between demands and resources is labeled with two figures denoting the perceived values of the link by both nodes connected by the link.

We can see that the order 1 is satisfied with the allocation of resources. Order 2 provides for less satisfied and not satisfied agents a new opportunity to improve their allocation. Many negotiation processes will begin which are proactively initiated by resources that aim to improve their allocations.

This increased activity of agents, sensing new opportunities combined with their dissatisfaction with the decision by the truck agent to accept new cargoes and create new journeys, may result in a ripple effect of changes to the schedule, accelerating the rate of change and causing a full collapse of the previously agreed schedule and its immediate re-building in a new manner. The schedule thus passes through a slowdown, accelerated activity, collapse into chaos and re-birth, a process known as 'constructive destruction'.

\subsection{Improving deteriorations}

In cargo transportation simulation system [15], orders are executed with a possible delay (penalties); transportation cost of each truck per time unit is the same. Unavailability period is simulated for one

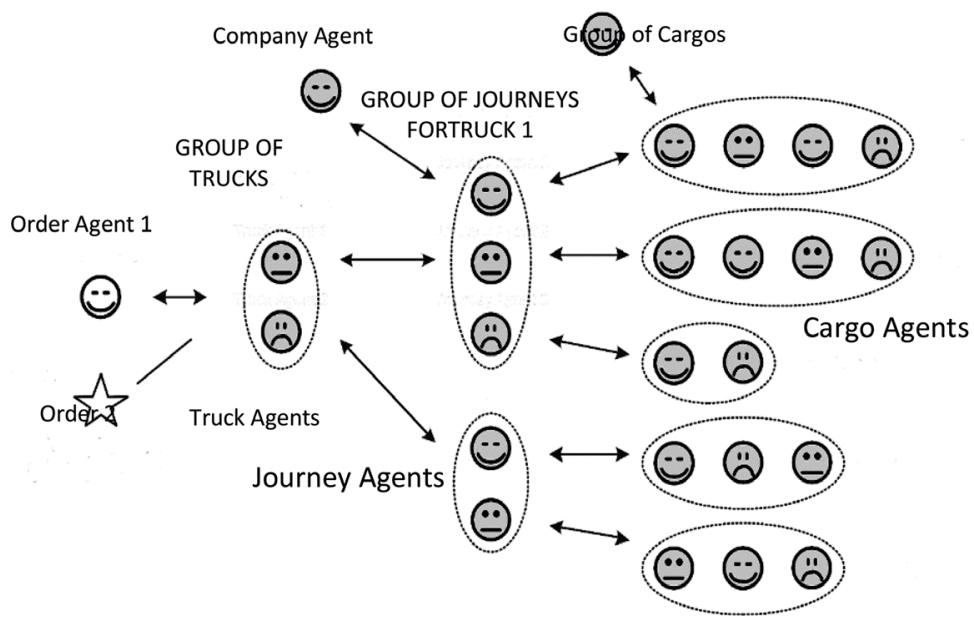

Figure 3: An example of autocatalytic reactions in the scheduler. 
of the trucks (e.g. the truck is not taking orders). The simulation was performed to determine the total profit of the system, including penalties and empty runs of trucks to the orders loading points (Fig. 4).

The average profit decreased; however, in the 30-day period of unavailability, a 5\% growth was observed, that is, 'situation deterioration' in this case led to more successful non-equilibrium state of the schedule, which is associated with a more profitable orders allocation to the remaining trucks. This phenomenon is similar to the well-known Braess's paradox [16]. In our case, adding trucks' working hours worsens the results and the introduction of restrictions improves it. In this case, adding a new resource worsens the results and the introduction of restrictions improves it. A multi-agent cargo transportation system increased its profit at some time period with decreasing part of the resources by self-organization and rescheduling.

\subsection{Loss of causality}

The initial events that lead to the rescheduling activate order and resource agents. Branching networks of changes do not allow identifying an event or an agent that caused the changes. Therefore, in time, the agents forget in what stages if the rescheduling they were involved. Waves of disturbance in the system activate the branching processes that do not contain the initial information about the cause of changes.

There were from two to six rescheduling times during the simulation of the loading distribution on the executing elements even with a small number of tasks (Fig. 5).

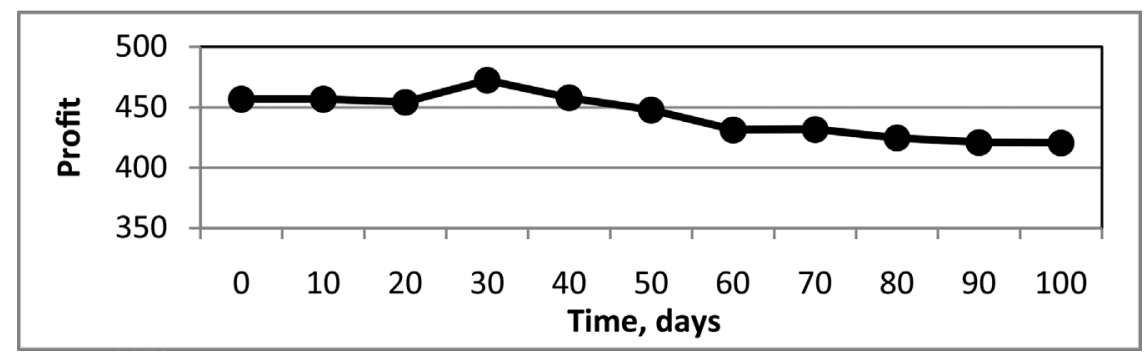

Figure 4: Transportation system total profit depending on the unavailability time of one of the trucks.

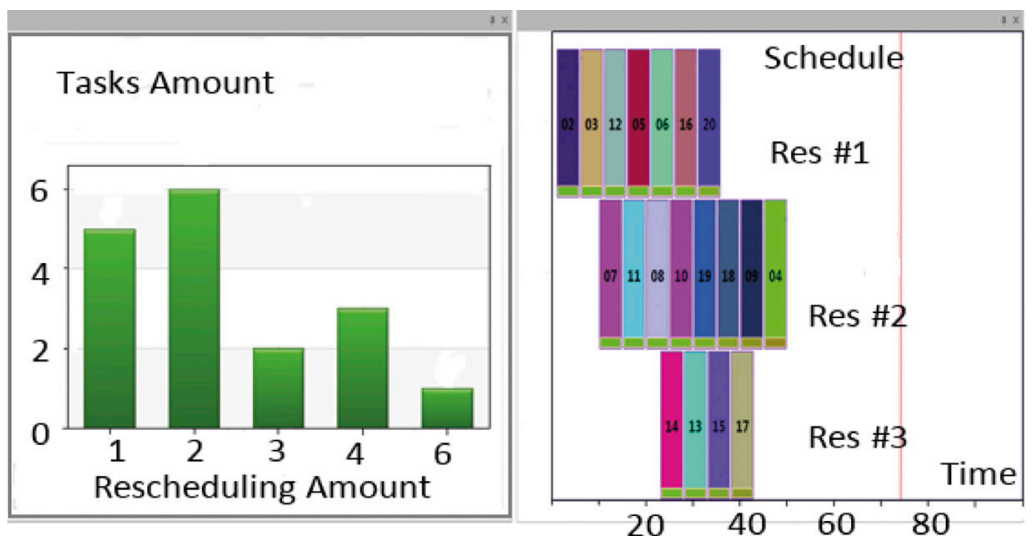

Figure 5: A bar chart of the system rescheduling (3 resources, 20 incoming tasks). 


\section{CONCLUSIONS}

Using the proposed DRN model, it will be possible to examine in detail in the future research the dynamics of self-organization processes, depending on the external incoming events, changes in the resources availability and operation in the condition of the changing microeconomics of agents system.

Metrics for the complexity parameters evaluation of the DRN will allow to study the dependency of adaptive properties of agents' self-organization from the dynamic structure of the network and to predict its response to external influences.

Dynamics model will provide an opportunity to study the processes of development and deterioration of the schedules over time as a phenomenon similar to the non-equilibrium thermodynamics.

\section{ACKNOWLEDGEMENT}

The study was supported by the Ministry of Education and Science of the Russian Federation.

\section{REFERENCES}

[1] Skobelev, P., Vittikh, V. Models of Self-organization for Designing Demand-Resource Networks, Automation and Remote Control. Journal of Russian Academy of Science, (1), pp. 177-185, 2003.

[2] Vittikh, V., Skobelev, P. The compensation method of agents interactions for real time resource allocation, Avtometriya. Journal of Siberian Branch of Russian Academy of Science, (2), pp. 78-87, 2009.

[3] Prigogine, I., Stengers, I. Order out of Chaos: Man's new dialogue with nature. Flamingo, 1984.

[4] Prigogine, I., Nicolis, G. Self-Organization in Non-Equilibrium Systems. Wiley. 1977.

[5] Leung, Y.-T. Handbook of Scheduling: Algorithms, Models and Performance Analysis, CRC Computer and Information Science Series, Chapman \& Hall, London, 2004.

[6] Shirzadeh Chaleshtari, A., Shadrokh, S. A Branch and Bound Algorithm for Resource Constrained Project Scheduling Problem subject to Cumulative Resources, World Academy of Science, Engineering and Technology, 6, pp. 23-28, 2012.

[7] Wooldridge, M. An Introduction to Multi-Agent Systems, John Wiley \& Sons, London, 2002.

[8] Shoham, Y., Leyton-Brown, K. Multiagent Systems: Algorithmic, Game-Theoretic, and Logical Foundations. Cambridge University Press, New York, 2009.

[9] Pechoucek, M., Marík., V. Industrial deployment of multi-agent technologies: review and selected case studies. Autonomous Agents and Multi-Agent Systems, 17(3), pp. 397-431, 2008. doi: http://dx.doi.org/10.1007/s10458-008-9050-0

[10] Leitao, P., Vrba, P. Recent Developments and Future Trends of Industrial Agents, Proceedings of the 5th International Conference on Holonic and Multi-Agent Systems in Manufacturing, Springer, Berlin, pp. 15-28, 2011. doi: http://dx.doi.org/10.1007/978-3-642-23181-0_2

[11] Brussel, H.V., Wyns, J., Valckenaers, P., Bongaerts, L. Reference architecture for holonic manufacturing systems: PROSA. Computer in Industry, 37(3), pp. 255-274, 1998. doi: http://dx.doi.org/10.1016/S0166-3615(98)00102-X

[12] Skobelev, P. Multi-Agent Systems for Real Time Resource Allocation, Scheduling, Optimization and Controlling: Industrial Applications (Invited Talk). Proceedings of the 5th International Conference on Industrial Applications of Holonic and Multi-Agent Systems, 6867, pp. 1-14, 2011. doi: http://dx.doi.org/10.1007/978-3-642-23181-0_1

[13] Rzevski, G., Skobelev, P. Managing complexity. WIT Press, UK-USA, 2014. 
[14] Zadeh, L.A. On the definition of adaptivity. Proceedings of the IEEE, 51(3), pp. 469-470, 1963. doi: http://dx.doi.org/10.1109/PROC.1963.1852

[15] Granichin, O., Skobelev, P., Lada, A., Tsarev, A. Cargo transportation models analysis using multi-agent adaptive real-time truck scheduling system. Proceedings of the 5th International Conference on Agents and Artificial Intelligence (ICAART'2013), February 15-18, 2013, Barcelona, Spain. - SciTePress, Portugal, 2, pp. 244-249, 2013.

[16] Braess's paradox. [online] Available at: https://en.wikipedia.org/wiki/ Braess's_paradox [20 January 2015]. 